\title{
Some Relations Between the Riemann Zeta Function and the Generalized Bernoulli Polynomials of Level $m$
}

\author{
Yamilet Quintana $^{1 *}$ and Héctor Torres-Guzmán ${ }^{1}$ \\ ${ }^{1}$ Departamento de Matemáticas Puras y Aplicadas, Edificio Matemáticas y Sistemas (MYS), Apartado Postal: 89000, Caracas 1080 A, \\ Universidad Simón Bolivar, Venezuela \\ * Corresponding author
}

\section{Article Info}

Keywords: Bernoulli polynomials, Euler-Maclaurin quadrature formulae, generalized Bernoulli polynomials of level m, quadrature formula, Riemann zeta function.

2010 AMS: 65D32, 41A55, 65B15,

$33 F 05$.

Received: 5 August 2019

Accepted: 14 September 2019

Available online: 26 December 2019

\begin{abstract}
The main purpose of this paper is to show some relations between the Riemann zeta function and the generalized Bernoulli polynomials of level $m$. Our approach is based on the use of Fourier expansions for the periodic generalized Bernoulli functions of level $m$, as well as quadrature formulae of Euler-Maclaurin type. Some illustrative examples involving such relations are also given.
\end{abstract}

\section{Introduction}

Let $\zeta(s)$ be the Riemann zeta function defined by

$$
\zeta(s)=\sum_{n=1}^{\infty} \frac{1}{n^{s}}, \quad \Re(s)>1 .
$$

It is a classical result due to Riemann that $\zeta(s)$ can be analytically continued to a meromorphic function on the whole complex plane with the only pole at $s=1$, which is a simple pole with residue 1 . Also, if we consider the classical Bernoulli polynomials given by

$$
\frac{z e^{x z}}{e^{z}-1}=\sum_{n=0}^{\infty} B_{n}(x) \frac{z^{n}}{n !}, \quad|z|<2 \pi
$$

and the classical Bernoulli numbers, $B_{n}=B_{n}(0)$, for all $n \geq 0$, then it is well known the following relation between $\zeta(s)$ and the Bernoulli polynomials:

$$
\zeta(2 k)=\frac{(-1)^{k-1} \pi^{2 k} 2^{2 k-1}}{(2 k) !} B_{2 k}, \quad k \geq 1
$$

Euler's relation (1.1) provides an elegant formula for the explicit evaluation of $\zeta(2 k)$, which shows the arithmetical nature of $\zeta(2 k)$ (cf. eg., $[3,4,5,6])$. However, for the zeta values $\zeta(2 k+1)$ there is very little known information. For instance, in his paper of $1981 \mathrm{R}$. Apéry showed that $\zeta(3)$ is irrational, but for $k \geq 2$ the arithmetical nature of $\zeta(2 k+1)$ remains open (cf. [1, 3, 4, 5, 6, 7] and the references thereof). In this contribution we are interested in exploring similar relations to (1.1) in the setting of the generalized Bernoulli polynomials of level $m$ $[15,18]$. In order to do that, we show some constraints of the use of Fourier expansions for the periodic generalized Bernoulli functions of level $m$, as well as, our approach which is mainly based on quadrature formulae of Euler-Maclaurin type. 
The outline of the paper as follows. Section 2 provides a short background about some relevant properties of the generalized Bernoulli polynomials of level $\mathrm{m}$. Section 3 is devoted to show some constraints of the use of Fourier expansions for the periodic generalized Bernoulli functions of level $m$ (see Theorems 3.2 and 3.3). Finally, Section 4 contains the basic ideas in order to obtain quadrature formulae of Euler-Maclaurin type based on generalized Bernoulli polynomials of level $m$ (see Theorem 4.2). Also, in this section is proved a result that reveals an interesting property about the applications of the quadrature formulae of Euler-Maclaurin type based on these polynomials (see Theorem 4.3). As usual, throughout this paper the convention $0^{0}=1$ will be adopted and an empty sum will be interpreted to be zero.

\section{Generalized Bernoulli polynomials of level $m$ : some properties}

For a fixed $m \in \mathbb{N}$, the generalized Bernoulli polynomials of level $m$ are defined by means of the following generating function $[14,15,18$, $20,21,22]$

$$
\frac{z^{m} e^{x z}}{e^{z}-\sum_{l=0}^{m-1} \frac{z^{l}}{l !}}=\sum_{n=0}^{\infty} B_{n}^{[m-1]}(x) \frac{z^{n}}{n !}, \quad|z|<2 \pi
$$

and, the generalized Bernoulli numbers of level $m$ are defined by $B_{n}^{[m-1]}:=B_{n}^{[m-1]}(0)$, for all $n \geq 0$. The generalized Bernoulli polynomials of level $m$ also have been called hypergeometric Bernoulli polynomials [12]. It is clear that if $m=1$ in (2.1), then we obtain the definition of the classical Bernoulli polynomials $B_{n}(x)$, and classical Bernoulli numbers, respectively, i.e., $B_{n}(x)=B_{n}^{[0]}(x)$, and $B_{n}=B_{n}^{[0]}$, respectively, for all $n \geq 0$.

It is not difficult to check that the first four generalized Bernoulli polynomials of level $m$ are:

$$
\begin{aligned}
& B_{0}^{[m-1]}(x)=m ! \\
& B_{1}^{[m-1]}(x)=m !\left(x-\frac{1}{m+1}\right) \\
& B_{2}^{[m-1]}(x)=m !\left(x^{2}-\frac{2}{m+1} x+\frac{2}{(m+1)^{2}(m+2)}\right), \\
& B_{3}^{[m-1]}(x)=m !\left(x^{3}-\frac{3}{m+1} x^{2}+\frac{6}{(m+1)^{2}(m+2)} x+\frac{6(m-1)}{(m+1)^{3}(m+2)(m+3)}\right) .
\end{aligned}
$$

The following results summarize some properties of the generalized Bernoulli polynomials of level $m$ (cf. [14, 15, 13, 18]).

Proposition 2.1. [18, Proposition 1] For a fixed $m \in \mathbb{N}$, let $\left\{B_{n}^{[m-1]}(x)\right\}_{n \geq 0}$ be the sequence of generalized Bernoulli polynomials of level m. Then the following statements hold:

a) Summation formula. For every $n \geq 0$,

$$
B_{n}^{[m-1]}(x)=\sum_{k=0}^{n}\left(\begin{array}{l}
n \\
k
\end{array}\right) B_{k}^{[m-1]} x^{n-k} .
$$

b) Differential relations (Appell polynomial sequences). For $n, j \geq 0$ with $0 \leq j \leq n$, we have

$$
\left[B_{n}^{[m-1]}(x)\right]^{(j)}=\frac{n !}{(n-j) !} B_{n-j}^{[m-1]}(x) .
$$

c) Inversion formula. [15, Equation (2.6)] For every $n \geq 0$,

$$
x^{n}=\sum_{k=0}^{n}\left(\begin{array}{l}
n \\
k
\end{array}\right) \frac{k !}{(m+k) !} B_{n-k}^{[m-1]}(x) .
$$

d) Recurrence relation. [15, Lemma 3.2] For every $n \geq 1$,

$$
B_{n}^{[m-1]}(x)=\left(x-\frac{1}{m+1}\right) B_{n-1}^{[m-1]}(x)-\frac{1}{n(m-1) !} \sum_{k=0}^{n-2}\left(\begin{array}{l}
n \\
k
\end{array}\right) B_{n-k}^{[m-1]} B_{k}^{[m-1]}(x) .
$$

e) Integral formulas.

$$
\begin{aligned}
& \int_{x_{0}}^{x_{1}} B_{n}^{[m-1]}(x) d x=\frac{1}{n+1}\left[B_{n+1}^{[m-1]}\left(x_{1}\right)-B_{n+1}^{[m-1]}\left(x_{0}\right)\right]=\sum_{k=0}^{n} \frac{1}{n-k+1}\left(\begin{array}{l}
n \\
k
\end{array}\right) B_{k}^{[m-1]}\left(\left(x_{1}\right)^{n-k+1}-\left(x_{0}\right)^{n-k+1}\right) . \\
& B_{n}^{[m-1]}(x)=n \int_{0}^{x} B_{n-1}^{[m-1]}(t) d t+B_{n}^{[m-1]} .
\end{aligned}
$$

f) $\left[15\right.$, Theorem 3.1] Differential equation. For every $n \geq 1$, the polynomial $B_{n}^{[m-1]}(x)$ satisfies the following differential equation

$$
\frac{B_{n}^{[m-1]}}{n !} y^{(n)}+\frac{B_{n-1}^{[m-1]}}{(n-1) !} y^{(n-1)}+\cdots+\frac{B_{2}^{[m-1]}}{2 !} y^{\prime \prime}+(m-1) !\left(\frac{1}{m+1}-x\right) y^{\prime}+n(m-1) ! y=0 .
$$

If we denote by $\mathbb{P}_{n}$ the linear space of polynomials with real coefficients and degree less than or equal to $n$, then (2.4) implies that 
Proposition 2.2. [18, Proposition 2] For a fixed $m \in \mathbb{N}$ and each $n \geq 0$, the set $\left\{B_{0}^{[m-1]}(x), B_{1}^{[m-1]}(x), \ldots, B_{n}^{[m-1]}(x)\right\}$ is a basis for $\mathbb{P}_{n}$, i.e.,

$$
\mathbb{P}_{n}=\operatorname{spanB}_{0}^{[m-1]}(x), B_{1}^{[m-1]}(x), \ldots, B_{n}^{[m-1]}(x)
$$

We conclude this section showing in Figure 2.1 the plots of some generalized Bernoulli polynomials of level $\mathrm{m}$.

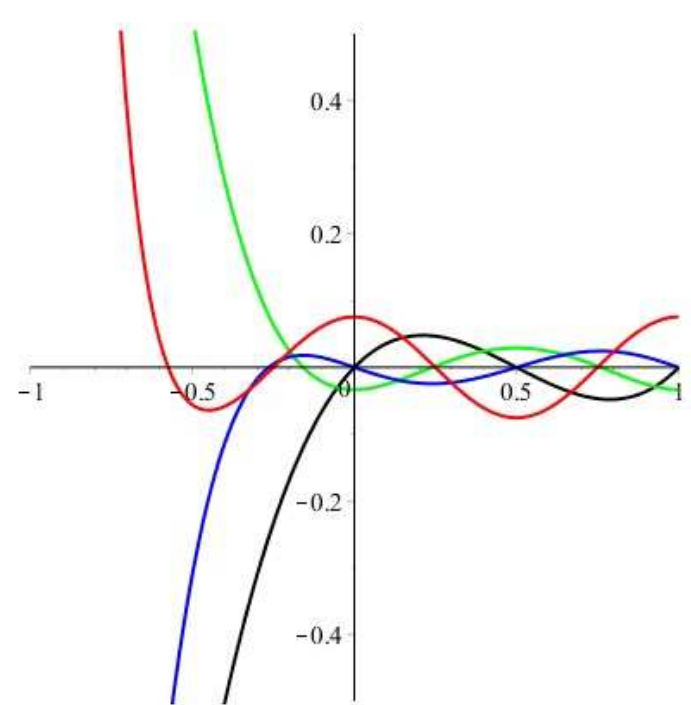

(a) Level: $m=1$. Degrees: $n=3$ (black), $n=4$ (green), $n=5$ (blue) $n=10$ (red).

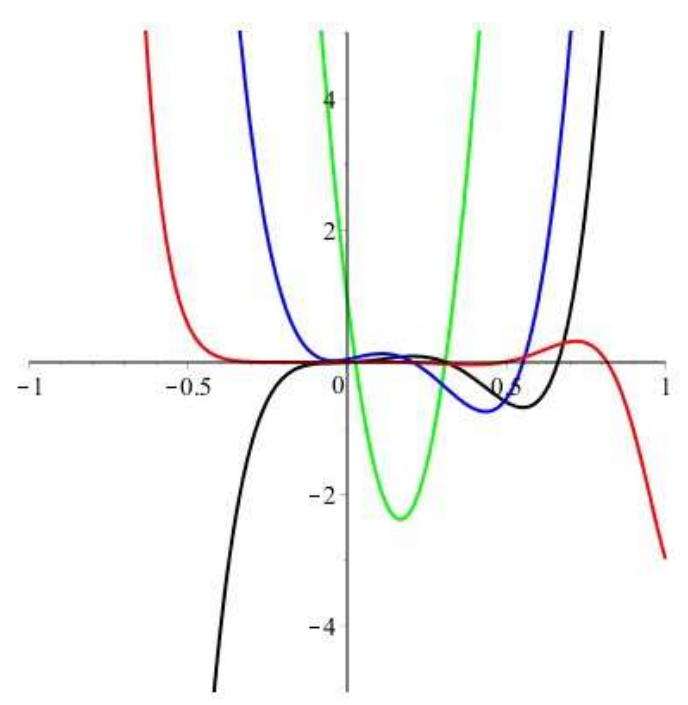

(b) Level: $m=5$. Degrees: $n=2$ (green), $n=5$ (blue), $n=6$ (black), $n=10$ (red).

Figure 2.1: Graphs of some generalized Bernoulli polynomials for the levels $m=1$ (classical Bernoulli polynomials) and $m=5$, respectively.

\section{Fourier expansions and generalized Bernoulli polynomials of level $m$}

For a fixed $m \in \mathbb{N}$, the periodic generalized Bernoulli functions of level $m$ are defined as follows.

$$
\begin{aligned}
& p_{n}^{[m-1]}(x)=\frac{B_{n}^{[m-1]}(x)}{n !}, \quad 0 \leq x<1, \\
& p_{n}^{[m-1]}(x+1)=p_{n}^{[m-1]}(x), \quad x \in \mathbb{R} .
\end{aligned}
$$

The functions $p_{n}^{[m-1]}(x)$ are continuous on $\mathbb{R}$ with continuous derivatives up to order $n-1$ only if $m=1$ and $n>2$.

In what follows, the symbol " " is used to refer to the formal Fourier expansion for a given function on an interval, and it is not associated to some notion of convergence in particular, since as we know there are several kinds of convergence involved with the notion of Fourier expansion associated to a given function.

For $m=1$ the Fourier expansions for the periodic generalized Bernoulli functions of level $m$ coincide with the Fourier expansions for the periodic Bernoulli functions, i.e.,

$$
\begin{gathered}
p_{1}^{[0]}(x)=p_{1}(x) \sim-\sum_{k=1}^{\infty} \frac{2 \sin (2 \pi k x)}{2 \pi k}, \\
p_{2 r}^{[0]}(x)=p_{2 r}(x)=(-1)^{r-1} \sum_{k=1}^{\infty} \frac{2 \cos (2 \pi k x)}{(2 \pi k)^{2 r}}, \\
p_{2 r+1}^{[0]}(x)=p_{2 r+1}(x)=(-1)^{r-1} \sum_{k=1}^{\infty} \frac{2 \sin (2 \pi k x)}{(2 \pi k)^{2 r+1}},
\end{gathered}
$$

with $r \geq 1$.

Notice that by a well known result on the uniform convergence of Fourier expansions (see, for instance, [10, 17, 23]), the Fourier series (3.3) and (3.4) are uniformly convergent, while this does not hold for the Fourier expansion (3.2), since

$$
p_{1}(0)=p_{1}(1)=-\frac{1}{2}, \quad \text { and } \quad \lim _{\varepsilon \rightarrow 0^{+}} p_{1}(1-\varepsilon)=\frac{1}{2}
$$

whereas the Fourier expansion (3.2) assumes the value 0 at both $x=0$ and $x=1$. Figure 3.1 shows the plots for some periodic Bernoulli functions. 


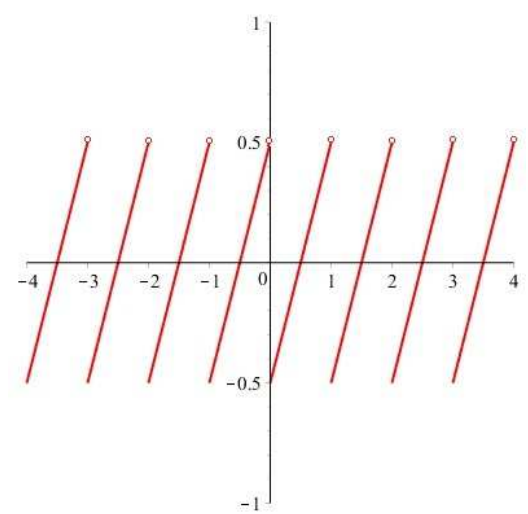

(a) Graph of $p_{1}(x)$.

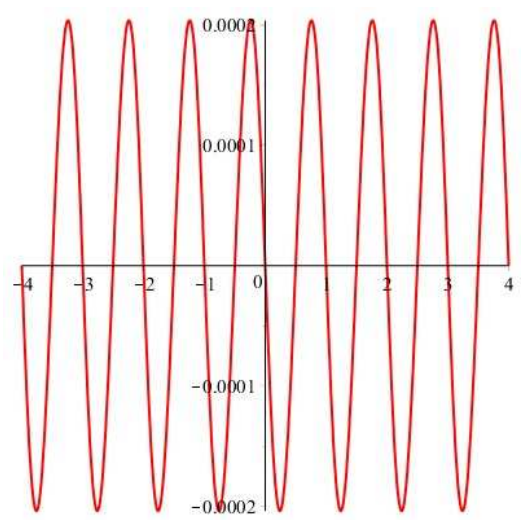

(b) Graph of $p_{5}(x)$.

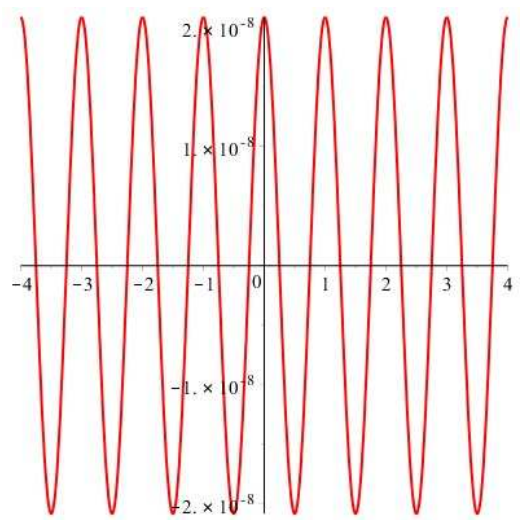

(c) Graph of $p_{10}(x)$.

Figure 3.1: Periodic Bernoulli functions for $n=1,5,10$.

It is important to note that the sequence of functions $\left\{p_{n}(x)\right\}_{n>2} \subset C^{n-2}(-\infty, \infty)$ (when $n=2$, we are using the notation $C^{0}(-\infty, \infty)=$ $C(-\infty, \infty)$ ), because the Bernoulli numbers satisfy the equality $B_{n}=(-1)^{n} B_{n}(1)$, for any $n \geq 0$ (see e.g., [3, Proposition 4.9]), $B_{n}=0$, if $n \geq 3$ is odd, and by the condition of periodicity (3.1) with $m=1$. In Figure 3.2 the plots for several generalized Bernoulli polynomials of level $m=5$ and their corresponding periodic generalized Bernoulli functions are shown.

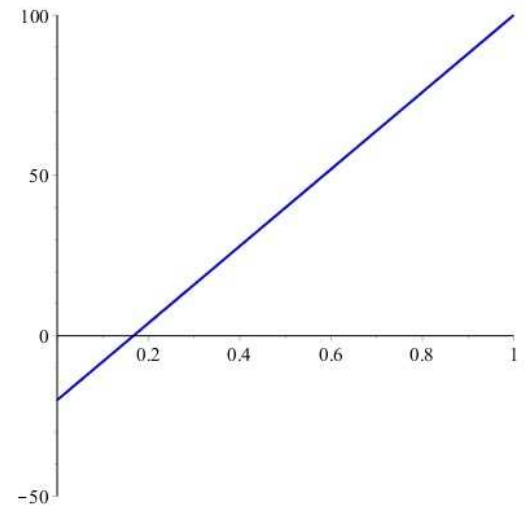

(a) Graph of $B_{1}^{[4]}(x)$.

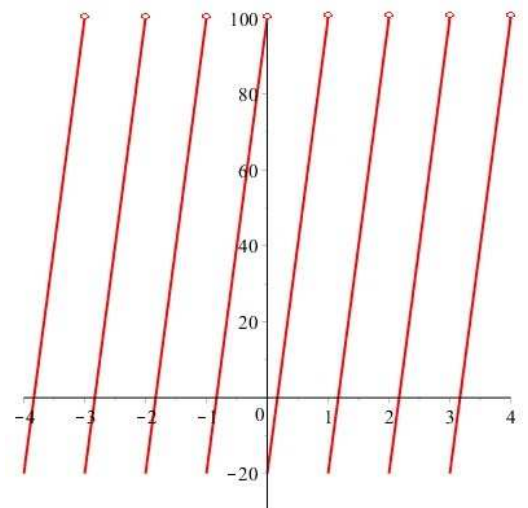

(b) Graph of $p_{1}^{[4]}(x)$.

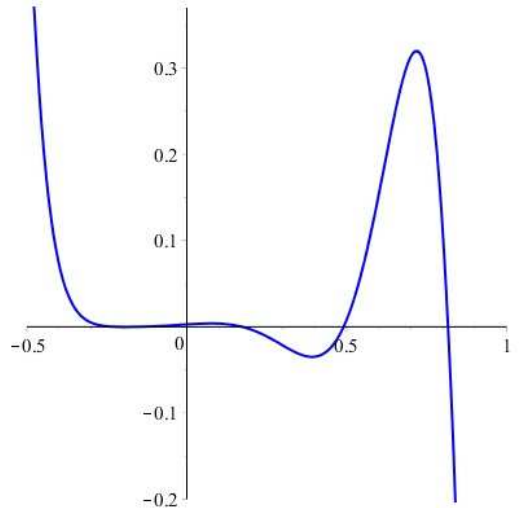

(c) Graph of $B_{10}^{[4]}(x)$.

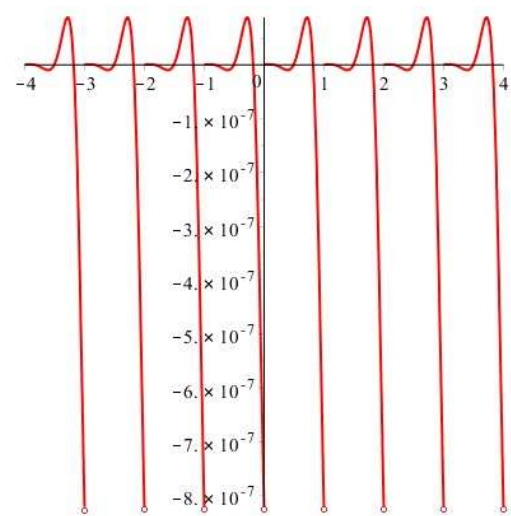

(d) Graph of $p_{10}^{[4]}(x)$.

Figure 3.2: Generalized Bernoulli polynomials of level $m=5$ and their corresponding periodic generalized Bernoulli functions for $n=1,10$.

It is worthy to mention that for $m, n>1$ the functions $p_{n}^{[m-1]}(x)$ are only differentiable on $\mathbb{R} \backslash \mathbb{Z}$-unlike what happens when $m=1$ and $n>2$ are considered (cf. [17, Chap. 3, Sec. 3.2])-. Thus, from (3.1) and (2.3) we deduce that $\left[p_{n+1}^{[m-1]}(x)\right]^{\prime}=p_{n}^{[m-1]}(x)$ for each $x \in(k, k+1)$, $k \in \mathbb{Z}$. Hence,

$$
\left[p_{n+1}^{[m-1]}(x)\right]^{\prime}=p_{n}^{[m-1]}(x), \quad \text { if } \quad x \in \mathbb{R} \backslash \mathbb{Z} .
$$

Also, the periodic generalized Bernoulli functions of level $m$ are integrable function on $[0,1]$. Therefore, they satisfy Dirichlet conditions for the existence of their Fourier expansions [10, 23]. 
For a fixed $m \in \mathbb{N}$ we note that $p_{1}^{[m-1]}(x)$ has the following Fourier coefficients:

$$
\begin{aligned}
& a_{0,1}^{[m-1]}=2 \int_{0}^{1} p_{1}^{[m-1]}(x) d x=\frac{m !}{2}\left(\frac{m-1}{m+1}\right), \\
& a_{k, 1}^{[m-1]}=2 \int_{0}^{1} p_{1}^{[m-1]}(x) \cos (2 \pi k x) d x=0, \\
& b_{k, 1}^{[m-1]}=2 \int_{0}^{1} p_{1}^{[m-1]}(x) \sin (2 \pi k x) d x=-\frac{2 m !}{2 \pi k},
\end{aligned}
$$

with $k \geq 1$. Thus, $p_{1}^{[m-1]}(x)$ has the Fourier expansion

$$
p_{1}^{[m-1]}(x) \sim \frac{m !}{2}\left(\frac{m-1}{m+1}\right)-\sum_{k=1}^{\infty} \frac{2 m ! \sin (2 \pi k x)}{2 \pi k} .
$$

For $x \in(0,1)$, let us integrate the series (3.6) formally, term by term:

$$
\begin{aligned}
\int_{0}^{x} p_{1}^{[m-1]}(t) d t & =\frac{m !}{2}\left(\frac{m-1}{m+1}\right) x-\sum_{k=1}^{\infty} \frac{2 m !}{2 \pi k} \int_{0}^{x} \sin (2 \pi k t) d t \\
& =\frac{m !}{2}\left(\frac{m-1}{m+1}\right) x-\sum_{k=1}^{\infty} \frac{2 m !}{(2 \pi k)^{2}}(1-\cos (2 \pi k x)) \\
& =\frac{m !}{2}\left(\frac{m-1}{m+1}\right) x-\frac{m !}{2 \pi^{2}} \zeta(2)+\sum_{k=1}^{\infty} \frac{2 m ! \cos (2 \pi k x)}{(2 \pi k)^{2}} .
\end{aligned}
$$

From (2.5) we have

$$
\int_{0}^{x} p_{1}^{[m-1]}(t) d t=p_{2}^{[m-1]}(x)-\frac{B_{2}^{[m-1]}}{2} .
$$

Hence, the substitution of (3.8) into (3.7) yields the following expansion for $p_{2}^{[m-1]}(x)$

$$
p_{2}^{[m-1]}(x)=\frac{B_{2}^{[m-1]}}{2}+\frac{m !}{2}\left(\frac{m-1}{m+1}\right) x-\frac{m !}{2 \pi^{2}} \zeta(2)+\sum_{k=1}^{\infty} \frac{2 m ! \cos (2 \pi k x)}{(2 \pi k)^{2}} .
$$

Since, $p_{2}^{[m-1]}(x)$ has the following Fourier coefficients:

$$
\begin{aligned}
& a_{0,2}^{[m-1]}=\frac{m !}{3}\left(\frac{m-1}{(m+1)^{2}}\right)\left(\frac{m^{2}+2 m-2}{m+2}\right), \\
& a_{k, 2}^{[m-1]}=2 \int_{0}^{1} p_{2}^{[m-1]}(x) \cos (2 \pi k x) d x=\frac{2 m !}{(2 \pi k)^{2}}, \\
& b_{k, 2}^{[m-1]}=2 \int_{0}^{1} p_{1}^{[m-1]}(x) \sin (2 \pi k x) d x=-\frac{m !}{2 \pi k}\left(\frac{m-1}{m+1}\right),
\end{aligned}
$$

with $k \geq 1$, then $p_{2}^{[m-1]}(x)$ has the Fourier expansion

$$
p_{2}^{[m-1]}(x)=\frac{m !}{6}\left(\frac{m-1}{(m+1)^{2}}\right)\left(\frac{m^{2}+2 m-2}{m+2}\right)+\sum_{k=1}^{\infty} \frac{2 m ! \cos (2 \pi k x)}{(2 \pi k)^{2}}-\sum_{k=1}^{\infty} \frac{m !(m-1) \sin (2 \pi k x)}{2 \pi k(m+1)} .
$$

On comparing (3.9) and (3.10), for $x \in(0,1)$ we see that

$$
\frac{m !}{2 \pi^{2}} \zeta(2)-\frac{B_{2}^{[m-1]}}{2}+\frac{m !}{6}\left(\frac{m-1}{(m+1)^{2}}\right)\left(\frac{m^{2}+2 m-2}{m+2}\right)=\frac{m !}{2}\left(\frac{m-1}{m+1}\right) x+\sum_{k=1}^{\infty} \frac{m !(m-1) \sin (2 \pi k x)}{2 \pi k(m+1)} .
$$

If we put $x=\frac{1}{2}$ in (3.11), then we obtain

$$
\zeta(2)=\frac{2 \pi^{2}}{m !}\left[\frac{B_{2}^{[m-1]}}{2}+\frac{m !}{4}\left(\frac{m-1}{m+1}\right)-\frac{m !}{6}\left(\frac{m-1}{(m+1)^{2}}\right)\left(\frac{m^{2}+2 m-2}{m+2}\right)\right] .
$$

The relation (3.12) connects the zeta number $\zeta(2)$ with the generalized Bernoulli polynomial $B_{2}^{[m-1]}(x)$ for any $m>1$. Notice that if $m=1$ then (3.12) coincides with Euler's relation (1.1) for $k=1$.

For example, if we take $m=2$ then (3.12) becomes

$$
\zeta(2)=\pi^{2}\left(\frac{B_{2}^{[1]}}{2}+\frac{1}{6}-\frac{1}{18}\right)=\frac{\pi^{2}}{6} .
$$


Since on $[0,1]$, the polynomial $p_{n}(x)$ is symmetric about the midpoint $x=\frac{1}{2}$, when $n$ is even, and it is antisymmetric about $x=\frac{1}{2}$, when $n$ is odd; that is,

$$
p_{n}(1-x)=(-1)^{n} p_{n}(x), \quad 0 \leq x \leq 1, \quad n \geq 2 .
$$

It follows that when $m=1$, taking $x=0$ in (3.3) and evaluating $p_{2 r}(0)$ from (3.1) and using (3.13), we obtain (cf. [17, Eq. (3.54)]):

$$
\zeta(2 r)=\sum_{n=1}^{\infty} \frac{1}{n^{2 r}}=(-1)^{r-1} \pi^{2 r} 2^{2 r-1} \frac{B_{2 r}}{(2 r) !}, \quad r \geq 1,
$$

this last equation is precisely (1.1).

Next, we will use the notation $p_{n}^{[m-1]}\left(x^{-}\right)$and $p_{n}^{[m-1]}\left(x^{+}\right)$for representing the one-sided limits $\lim _{y \rightarrow x^{-}} p_{n}^{[m-1]}(y)$ and $\lim _{y \rightarrow x^{+}} p_{n}^{[m-1]}(y)$, respectively. The following Proposition provides the Fourier expansion for $p_{n}^{[m-1]}(x)$ when $m>1$.

Proposition 3.1. For a fixed $m \in \mathbb{N}$ and any $n \in \mathbb{N}$, let $p_{n}^{[m-1]}(x)$ be the periodic generalized Bernoulli functions of level $m$. Then Fourier expansion for $p_{n}^{[m-1]}(x)$ on $[0,1]$ is given by

$$
p_{n}^{[m-1]}(x) \sim \frac{a_{0, n}^{[m-1]}}{2}+\sum_{k=1}^{\infty} a_{k, n}^{[m-1]} \cos (2 \pi k x)+\sum_{k=1}^{\infty} b_{k, n}^{[m-1]} \sin (2 \pi k x),
$$

where

$$
\frac{a_{0, n}^{[m-1]}}{2}=p_{n+1}^{[m-1]}\left(1^{-}\right)-p_{n+1}^{[m-1]}(0)=\frac{1}{(n+1) !}\left(B_{n+1}^{[m-1]}(1)-B_{n+1}^{[m-1]}\right) .
$$

And for $k \geq 1$ :

$$
\begin{aligned}
a_{k, n}^{[m-1]} & =\sum_{j=0}^{\left\lfloor\frac{n}{2}\right\rfloor-1}(-1)^{j} \frac{2}{(2 \pi k)^{2 j+2}}\left(p_{n-2 j-1}^{[m-1]}\left(1^{-}\right)-p_{n-2 j-1}^{[m-1]}(0)\right) \\
& =\sum_{j=0}^{\left\lfloor\frac{n}{2}\right\rfloor-1}(-1)^{j} \frac{2}{(2 \pi k)^{2 j+2}} \frac{\left(B_{n-2 j-1}^{[m-1]}(1)-B_{n-2 j-1}^{[m-1]}\right)}{(n-2 j-1) !}, \\
b_{k, n}^{[m-1]} & =\sum_{j=0}^{\left\lfloor\frac{n}{2}\right\rfloor}(-1)^{j+1} \frac{2}{(2 \pi k)^{2 j+1}}\left(p_{n-2 j}^{[m-1]}\left(1^{-}\right)-p_{n-2 j}^{[m-1]}(0)\right) \\
& =\sum_{j=0}^{\left\lfloor\frac{n}{2}\right\rfloor}(-1)^{j+1} \frac{2}{(2 \pi k)^{2 j+1}} \frac{\left(B_{n-2 j}^{[m-1]}(1)-B_{n-2 j}^{[m-1]}\right)}{(n-2 j) !} .
\end{aligned}
$$

Proof. For each $p_{n}^{[m-1]}(x)$ it is well known that its Fourier coefficients are given by

$$
\begin{aligned}
& a_{0, n}^{[m-1]}=2 \int_{0}^{1} p_{n}^{[m-1]}(x) d x, \\
& a_{k, n}^{[m-1]}=2 \int_{0}^{1} p_{n}^{[m-1]}(x) \cos (2 \pi k x) d x, \\
& b_{k, n}^{[m-1]}=2 \int_{0}^{1} p_{n}^{[m-1]}(x) \sin (2 \pi k x) d x,
\end{aligned}
$$

with $k \geq 1$. Then, (3.15) is a straightforward consequence of (3.20) and (3.5). For obtaining the relations (3.16) and (3.18) it suffices use integration by parts on the right-hand side of (3.21) and (3.22), respectively. So, we get

$$
\begin{aligned}
a_{k, n}^{[m-1]} & =-\frac{1}{2 \pi k} b_{k, n-1}^{[m-1]}, \\
b_{k, n}^{[m-1]} & =-\frac{2}{2 \pi k}\left(p_{n}^{[m-1]}\left(1^{-}\right)-p_{n}^{[m-1]}(0)\right)+\frac{1}{2 \pi k} a_{k, n-1}^{[m-1]} .
\end{aligned}
$$

Then replacing $n$ by $n-1$ in (3.24) and substituting the result obtained into (3.23), we get the following recurrence relation

$$
a_{k, n}^{[m-1]}+\frac{1}{(2 \pi k)^{2}} a_{k, n-2}^{[m-1]}=\frac{2}{(2 \pi k)^{2}}\left(p_{n-1}^{[m-1]}\left(1^{-}\right)-p_{n-1}^{[m-1]}(0)\right)
$$

Analogously, we can obtain

$$
b_{k, n}^{[m-1]}+\frac{1}{(2 \pi k)^{2}} b_{k, n-2}^{[m-1]}=-\frac{2}{2 \pi k}\left(p_{n}^{[m-1]}\left(1^{-}\right)-p_{n}^{[m-1]}(0)\right) .
$$


Finally, it follows from (3.25) and (3.26) that

$$
\begin{aligned}
a_{k, n}^{[m-1]} & =\frac{2}{(2 \pi k)^{2}}\left(p_{n-1}^{[m-1]}\left(1^{-}\right)-p_{n-1}^{[m-1]}(0)\right)-\frac{2}{(2 \pi k)^{4}}\left(p_{n-3}^{[m-1]}\left(1^{-}\right)-p_{n-3}^{[m-1]}(0)\right)+\frac{2}{(2 \pi k)^{6}}\left(p_{n-5}^{[m-1]}\left(1^{-}\right)-p_{n-5}^{[m-1]}(0)\right) \\
& -\frac{2}{(2 \pi k)^{8}}\left(p_{n-7}^{[m-1]}\left(1^{-}\right)-p_{n-7}^{[m-1]}(0)\right)+\cdots+(-1)^{\left\lfloor\frac{n}{2}\right\rfloor-1} \frac{2}{(2 \pi k)^{2\left\lfloor\frac{n}{2}\right\rfloor}}\left(p_{n-\left(\left\lfloor\frac{n}{2}\right\rfloor-1\right)}^{[m-1]}\left(1^{-}\right)-p_{n-\left(\left\lfloor\frac{n}{2}\right\rfloor-1\right)}^{[m-1]}(0)\right)
\end{aligned}
$$

and

$$
\begin{aligned}
b_{k, n}^{[m-1]} & =-\frac{2}{2 \pi k}\left(p_{n}^{[m-1]}\left(1^{-}\right)-p_{n}^{[m-1]}(0)\right)+\frac{2}{(2 \pi k)^{3}}\left(p_{n-2}^{[m-1]}\left(1^{-}\right)-p_{n-2}^{[m-1]}(0)\right)-\frac{2}{(2 \pi k)^{5}}\left(p_{n-4}^{[m-1]}\left(1^{-}\right)-p_{n-4}^{[m-1]}(0)\right) \\
& +\frac{2}{(2 \pi k)^{7}}\left(p_{n-6}^{[m-1]}\left(1^{-}\right)-p_{n-6}^{[m-1]}(0)\right)+\cdots+(-1)^{\left\lfloor\frac{n}{2}\right\rfloor+1} \frac{2}{(2 \pi k)^{2\left\lfloor\frac{n}{2}\right\rfloor+1}}\left(p_{n-2\left\lfloor\frac{n}{2}\right\rfloor}^{[m-1]}\left(1^{-}\right)-p_{n-2\left\lfloor\frac{n}{2}\right\rfloor}^{[m-1]}(0)\right) .
\end{aligned}
$$

From these last relations we obtain (3.17) and (3.19), respectively.

Theorem 3.2. For a fixed $m \in \mathbb{N}$ and $n \in \mathbb{N}$, let $p_{n}^{[m-1]}(x)$ be the periodic generalized Bernoulli functions of level $m$. If $x \in(0,1)$, then the following identity holds.

$$
\begin{aligned}
p_{n}^{[m-1]}(x) & =\frac{m !(m-1)}{2(m+1)} \frac{x^{n}}{n !}+m ! p_{n}(x)+\sum_{k=1}^{\left\lfloor\frac{n}{2}\right\rfloor}\left(\begin{array}{c}
n \\
2 k
\end{array}\right)\left[(2 k) ! p_{2 k}^{[m-1]}(0)+\frac{2(-1)^{k} m !(2 k) ! \zeta(2 k)}{(2 \pi)^{2 k}}\right] \frac{x^{n-2 k}}{n !} \\
& +\sum_{k=1}^{\left\lfloor\frac{n-1}{2}\right\rfloor} p_{2 k-1}^{[m-1]}(0) \frac{x^{n-2 k-1}}{(n-2 k-1) !}
\end{aligned}
$$

Proof. Using Proposition 3.1 we obtain the following expression for $p_{1}^{[m-1]}(x)$ :

$$
p_{1}^{[m-1]}(x)=\frac{m !(m-1)}{2(m+1)}-m ! \sum_{k=1}^{\infty} \frac{2 \sin 2 \pi k x}{2 \pi k}, \quad \text { whenever } x \in(0,1) .
$$

Then in view of (3.1) and (2.6), we see that

$$
p_{n}^{[m-1]}(x)=p_{n}^{[m-1]}(0)+\int_{0}^{x} p_{n-1}^{[m-1]}(t) d t, \quad \text { if } x \in[0,1)
$$

Taking $n=2$ and substituting (3.28) into (3.29), we get

$$
p_{2}^{[m-1]}(x)=p_{2}^{[m-1]}(0)+\int_{0}^{x} p_{1}^{[m-1]}(t) d t=\frac{m !(m-1)}{2(m+1)} x+\left(2 ! p_{2}^{[m-1]}(0)-\frac{2 m ! 2 !}{(2 \pi)^{2}} \zeta(2)\right) \frac{1}{2 !}+m ! p_{2}(x) .
$$

Similarly, for $n=3$ we can deduce

$$
p_{3}^{[m-1]}(x)=p_{3}^{[m-1]}(0)+\int_{0}^{x} p_{2}^{[m-1]}(t) d t=\frac{m !(m-1)}{2(m+1)} \frac{x^{2}}{2 !}+\left(2 ! p_{2}^{[m-1]}(0)-\frac{2 m ! 2 !}{(2 \pi)^{2}} \zeta(2)\right) \frac{1}{2 !} x+p_{3}^{[m-1]}(0)+m ! p_{3}(x)
$$

Iterating this procedure (3.27) follows.

Recall that the Dirichlet convergence theorem [10, 17, 23] guarantees that the Fourier series (3.14) converges pointwise at $x \in \mathbb{Z}$ to the average of $p_{n}^{[m-1]}\left(x^{+}\right)$and $p_{n}^{[m-1]}\left(x^{-}\right)$. Indeed, based on this fact we prove the next result.

Theorem 3.3. For a fixed $m \in \mathbb{N}$ and any $r \in \mathbb{N}$, the following identity holds.

$$
\zeta(2 r)=\frac{(-1)^{r-1} 2^{2 r-1} \pi^{2 r} B_{2 r}^{[m-1]}}{m !(2 r) !}+\Delta_{r}^{[m-1]}
$$

where

$$
\begin{aligned}
\Delta_{r}^{[m-1]} & =\frac{(-1)^{r-1} 2^{2 r-1} \pi^{2 r}}{m !}\left[\frac{B_{2 r}^{[m-1]}(1)-B_{2 r}^{[m-1]}}{2(2 r) !}-\frac{B_{2 r+1}^{[m-1]}(1)-B_{2 r+1}^{[m-1]}}{(2 r+1) !}-\sum_{j=1}^{r-1} \frac{\left(B_{2 r-2 j+1}^{[m-1]}(1)-B_{2 r-2 j+1}^{[m-1]}\right.}{(2 r-2 j+1) !} \frac{B_{2 j}}{(2 j) !}\right] \\
& =\frac{(-1)^{r-1} 2^{2 r-1} \pi^{2 r}}{m !}\left[\frac{1}{2(2 r) !} \sum_{k=0}^{2 r-1}\left(\begin{array}{c}
2 r \\
k
\end{array}\right) B_{k}^{[m-1]}-\frac{1}{(2 r+1) !} \sum_{k=0}^{2 r}\left(\begin{array}{c}
2 r+1 \\
k
\end{array}\right) B_{k}^{[m-1]}-\sum_{j=1}^{r-1} \sum_{k=0}^{2 j}\left(\begin{array}{c}
2 j+1 \\
k
\end{array}\right) \frac{B_{k}^{[m-1]} B_{2 j}}{(2 j+1) !(2 j) !}\right] .
\end{aligned}
$$


Proof. Let us consider $n=2 r$ and $x=0$ in (3.14). Since $x=0$ is a point of discontinuity of $p_{2 r}^{[m-1]}(x)$, by the Dirichlet convergence theorem $[10,17,23]$ we have

$$
\frac{p_{2 r}^{[m-1]}\left(0^{+}\right)+p_{2 r}^{[m-1]}\left(0^{-}\right)}{2}=\frac{a_{0,2 r}^{[m-1]}}{2}+\sum_{k=1}^{\infty} a_{k, 2 r}^{[m-1]} .
$$

Since

$$
\frac{p_{2 r}^{[m-1]}\left(0^{+}\right)+p_{2 r}^{[m-1]}\left(0^{-}\right)}{2}=\frac{B_{2 r}^{[m-1]}+B_{2 r}^{[m-1]}(1)}{2(2 r) !}
$$

using (3.15) and (3.17), we can rewrite (3.33) as follows

$$
\frac{B_{2 r}^{[m-1]}+B_{2 r}^{[m-1]}(1)}{2(2 r) !}=\frac{1}{(2 r+1) !}\left(B_{2 r+1}^{[m-1]}(1)-B_{2 r+1}^{[m-1]}\right)+\sum_{k=1}^{\infty} \sum_{j=0}^{r-1}(-1)^{j} \frac{2}{(2 \pi k)^{2 j+2}} \frac{\left(B_{2 r-2 j-1}^{[m-1]}(1)-B_{2 r-2 j-1}^{[m-1]}\right)}{(2 r-2 j-1) !}
$$

Taking into account that

$$
p_{2 j+2}(0)=(-1)^{j+1} \sum_{n=1}^{\infty} \frac{2}{(2 \pi n)^{2 j+2}},
$$

the relation (3.34) can be expressed as

$$
\frac{B_{2 r}^{[m-1]}+B_{2 r}^{[m-1]}(1)}{2(2 r) !}=\frac{1}{(2 r+1) !}\left(B_{2 r+1}^{[m-1]}(1)-B_{2 r+1}^{[m-1]}\right)+m ! p_{2 r}(0)+\sum_{j=0}^{r-2} \frac{\left(B_{2 r-2 j-1}^{[m-1]}(1)-B_{2 r-2 j-1}^{[m-1]}\right)}{(2 r-2 j-1) !} \frac{B_{2 j+2}}{(2 j+2) !} .
$$

Or equivalently,

$$
\frac{B_{2 r}^{[m-1]}+B_{2 r}^{[m-1]}(1)}{2(2 r) !}=\frac{1}{(2 r+1) !}\left(B_{2 r+1}^{[m-1]}(1)-B_{2 r+1}^{[m-1]}\right)+m ! p_{2 r}(0)+\sum_{j=1}^{r-1} \frac{\left(B_{2 r-2 j+1}^{[m-1]}(1)-B_{2 r-2 j+1}^{[m-1]}\right)}{(2 r-2 j+1) !} \frac{B_{2 j}}{(2 j) !} .
$$

Now, from (3.35) we deduce that

$$
\frac{2(-1)^{r-1} \zeta(2 r)}{(2 \pi)^{2 r}}=\frac{B_{2 r}^{[m-1]}(1)+B_{2 r}^{[m-1]}}{2 m !(2 r) !}-\frac{B_{2 r+1}^{[m-1]}(1)-B_{2 r+1}^{[m-1]}}{m !(2 r+1) !}-\frac{1}{m !} \sum_{j=1}^{r-1} \frac{\left(B_{2 r-2 j+1}^{[m-1]}(1)-B_{2 r-2 j+1}^{[m-1]}\right)}{(2 r-2 j+1) !} \frac{B_{2 j}}{(2 j) !} .
$$

Hence, (3.36) takes the form:

$$
\zeta(2 r)=\frac{(-1)^{r-1} 2^{2 r-1} \pi^{2 r} B_{2 r}^{[m-1]}}{m !(2 r) !}+\Delta_{r}^{[m-1]},
$$

where

$$
\Delta_{r}^{[m-1]}=\frac{(-1)^{r-1} 2^{2 r-1} \pi^{2 r}}{m !}\left[\frac{B_{2 r}^{[m-1]}(1)+B_{2 r}^{[m-1]}}{2(2 r) !}-\frac{B_{2 r+1}^{[m-1]}(1)-B_{2 r+1}^{[m-1]}}{(2 r+1) !}-\sum_{j=1}^{r-1} \frac{\left(B_{2 r-2 j+1}^{[m-1]}(1)-B_{2 r-2 j+1}^{[m-1]}\right)}{(2 r-2 j+1) !} \frac{B_{2 j}}{(2 j) !}\right] .
$$

Hence, $\Delta_{r}^{[m-1]}$ satisfies (3.31).

Finally, the substitution of (2.2) into the above expression for $\Delta_{r}^{[m-1]}$, and some suitable computations yield the identity (3.32).

Notice that if $m=1$ in (3.30) then we recover (1.1). It is not difficult to see that for $r=1$ the identity (3.30) yields the same result than the identity (3.12).

\section{Riemann zeta function and quadrature formulae of Euler-Maclaurin type}

It is well known that using the Euler-Maclaurin summation formula (cf. [2, 8, 11], and [16, Chap. 2, Sec. 3, p. 30]) it is possible to deduce the following formula for the integral of the product of two classical Bernoulli polynomials

$$
\int_{0}^{1} B_{s}(t) B_{r}(t) d t=(-1)^{s+1} \frac{s ! r !}{(s+r) !} B_{s+r}, \quad \text { where } r, s \geq 1 .
$$

Using integration by parts a similar formula to (4.1) has been deduced in [18]. More precisely, for an integer $r \geq 0$ and a closed interval $[a, b]$, let $C^{r}[a, b]$ denote the set of all $r$-times continuously differentiable functions defined on $[a, b]$. Then following result holds. 
Lemma 4.1. [18, Lemma 1] Let $r \geq 1$ and $f \in C^{r}[0,1]$. For a fixed $m \in \mathbb{N}$, we have

$$
\int_{0}^{1} f(t) d t=\frac{1}{m !}\left[\sum_{k=1}^{r} A_{k}^{[m-1]}(f)+\frac{(-1)^{r}}{r !} \int_{0}^{1} f^{(r)}(t) B_{r}^{[m-1]}(t) d t\right],
$$

where

$$
A_{k}^{[m-1]}(f)=\frac{(-1)^{k}}{k !}\left(f^{(k-1)}(0) B_{k}^{[m-1]}-f^{(k-1)}(1) B_{k}^{[m-1]}(1)\right), \quad k=1, \ldots, r .
$$

Applying the substitution $f(t)=B_{r+n}^{[m-1]}(t)$ into (4.2) and taking into account (2.3), (2.6) we have

$$
\int_{0}^{1} B_{r}^{[m-1]}(t) B_{n}^{[m-1]}(t) d t=\frac{(-1)^{r+1} r ! n ! m !}{(r+n) !}\left[\frac{B_{r+n+1}^{[m-1]}-B_{r+n+1}^{[m-1]}(1)}{r+n+1}+\frac{1}{m !} \sum_{k=1}^{r} A_{k}^{[m-1]}\right],
$$

where $r, n \geq 1$ and

$$
A_{k}^{[m-1]}=\frac{(-1)^{k}}{k}\left(\begin{array}{c}
r+n \\
k-1
\end{array}\right)\left(B_{r+n-k+1}^{[m-1]} B_{k}^{[m-1]}-B_{r+n-k+1}^{[m-1]}(1) B_{k}^{[m-1]}(1)\right), \quad k=1, \ldots, r .
$$

The expression (4.3) is the analogue of (4.1) in the setting of the generalized Bernoulli polynomials of level $m$. We strongly recommend to the interested reader see [18] for the corresponding proofs of the results mentioned above.

Let $L^{2}[0,1]$ be the space of the square-integrable functions on $[0,1]$, endowed with the norm

$$
\|f\|_{L^{2}[0,1]}:=\left(\int_{0}^{1}|f(t)|^{2} d t\right)^{1 / 2}=\langle f, f\rangle^{1 / 2}
$$

where

$$
\langle f, g\rangle:=\int_{0}^{1} f(t) g(t) d t, \text { for every } f, g \in L^{2}[0,1] .
$$

It is not difficult to see that we can determine the norm $\left\|B_{n}^{[m-1]}\right\|_{L^{2}[0,1]}$ using (4.3), as

$$
\begin{aligned}
\left\|B_{n}^{[m-1]}\right\|_{L^{2}[0,1]}^{2} & =\frac{(n !)^{2} m !(-1)^{n}}{(2 n+1) !}\left(B_{2 n+1}^{[m-1]}(1)-B_{2 n+1}^{[m-1]}\right) \\
& +(n !)^{2}(-1)^{n+1} \sum_{k=1}^{n} \frac{(-1)^{k}}{(2 n+1-k) ! k !}\left(B_{2 n+1-k}^{[m-1]} B_{k}^{[m-1]}-B_{2 n+1-k}^{[m-1]}(1) B_{k}^{[m-1]}(1)\right) .
\end{aligned}
$$

From the trigonometric form of Fourier expansion for $f \in L^{2}[0,1]$ it is possible to deduce the following form of Parseval's identity:

$$
\|f\|_{L^{2}[0,1]}^{2}=\frac{\left|a_{0}(f)\right|^{2}}{4}+\frac{1}{2} \sum_{k=1}^{\infty}\left|a_{k}(f)\right|^{2}+\left|b_{k}(f)\right|^{2},
$$

where

$$
\begin{aligned}
& a_{k}(f)=2 \int_{0}^{1} f(x) \cos (2 \pi k x) d x, \quad k \geq 0, \\
& b_{k}(f)=2 \int_{0}^{1} f(x) \sin (2 \pi k x) d x, \quad k \geq 1 .
\end{aligned}
$$

Hence, using (4.4) we show how linear combinations of the values of $\zeta(2 k)$ can be obtained by applying Parseval's identity (4.5) with the Fourier coefficients (3.15), (3.17) and (3.19) of the periodic generalized Bernoulli functions of level $m$.

Applying Parseval's identity (4.5) to $p_{n}^{[m-1]}(x)$ and using (3.15)-(3.19), we can deduce that

$$
\begin{aligned}
\left\|B_{n}^{[m-1]}\right\|_{L^{2}[0,1]}^{2} & =(n !)^{2}\left[\frac{\left(a_{0, n}^{[m-1]}\right)^{2}}{4}+\frac{1}{2} \sum_{k=1}^{\infty}\left(a_{k, n}^{[m-1]}\right)^{2}+\left(b_{k, n}^{[m-1]}\right)^{2}\right] \\
& =\frac{\left(B_{n+1}^{[m-1]}(1)-B_{n+1}^{[m-1]}\right)^{2}}{(n+1)^{2}}+2(n !)^{2} \sum_{k=1}^{\infty}\left[\sum_{j=0}^{\left\lfloor\frac{n}{2}\right\rfloor-1} \frac{(-1)^{j}}{(2 \pi k)^{2 j+2}}\left(\frac{B_{n-2 j-1}^{[m-1]}(1)-B_{n-2 j-1}^{[m-1]}}{(n-2 j-1) !}\right)\right]^{2} \\
& +2(n !)^{2} \sum_{k=1}^{\infty}\left[\sum_{j=0}^{\left\lfloor\frac{n}{2}\right\rfloor} \frac{(-1)^{j+1}}{(2 \pi k)^{2 j+1}}\left(\frac{B_{n-2 j}^{[m-1]}(1)-B_{n-2 j}^{[m-1]}}{(n-2 j) !}\right)\right]^{2} .
\end{aligned}
$$

Comparing (4.4) with (4.6) we obtain the next equality: 


$$
\begin{aligned}
\sum_{k=1}^{\infty} A_{k, n}^{2}+B_{k, n}^{2} & =\frac{m !(-1)^{n}}{2(2 n+1) !}\left(B_{2 n+1}^{[m-1]}(1)-B_{2 n+1}^{[m-1]}\right)-\frac{\left(B_{n+1}^{[m-1]}(1)-B_{n+1}^{[m-1]}\right)^{2}}{2(n+1)^{2}} \\
& +\frac{(-1)^{n+1}}{2} \sum_{k=1}^{n} \frac{(-1)^{k}}{(2 n+1-k) ! k !}\left(B_{2 n+1-k}^{[m-1]} B_{k}^{[m-1]}-B_{2 n+1-k}^{[m-1]}(1) B_{k}^{[m-1]}(1)\right)
\end{aligned}
$$

where

$$
\begin{aligned}
A_{k, n} & =\sum_{j=0}^{\left\lfloor\frac{n}{2}\right\rfloor-1} \frac{(-1)^{j}}{(2 \pi k)^{2 j+2}}\left(\frac{B_{n-2 j-1}^{[m-1]}(1)-B_{n-2 j-1}^{[m-1]}}{(n-2 j-1) !}\right), \\
B_{k, n} & =\sum_{j=0}^{\left\lfloor\frac{n}{2}\right\rfloor} \frac{(-1)^{j+1}}{(2 \pi k)^{2 j+1}}\left(\frac{B_{n-2 j}^{[m-1]}(1)-B_{n-2 j}^{[m-1]}}{(n-2 j) !}\right) .
\end{aligned}
$$

Furthermore, if $m=1$ in (4.7) we recover (1.1). Following the ideas of [19] we can obtain a quadrature formulae of Euler-Maclaurin type based on generalized Bernoulli polynomials of level $m \in \mathbb{N} \backslash\{1\}$.

Theorem 4.2. Let $r \geq 1, f \in C^{r}[a, b]$ and $m \in \mathbb{N}$. For a fixed $n \in \mathbb{N}$ let $x_{j}=a+j h, j=0,1, \ldots, n$, where $h=\frac{b-a}{n}$, and $f_{j}^{(k-1)}=f^{(k-1)}\left(x_{j}\right)$, $k=1,2, \ldots, r$. Then, the following composite trapezoidal rules hold.

$$
\int_{a}^{b} f(t) d t=\sum_{j=0}^{n-1} \sum_{k=1}^{r} \tilde{A}_{k, j}^{[m-1]}(f)+R_{r}^{[m-1]}(f),
$$

where

$$
\tilde{A}_{k, j}^{[m-1]}(f)=\frac{(-1)^{k+1}}{m ! k !} h^{k}\left(f_{j+1}^{(k-1)} B_{k}^{[m-1]}(1)-f_{j}^{(k-1)} B_{k}^{[m-1]}\right), \quad 1 \leq k \leq r
$$

and

$$
R_{r}^{[m-1]}(f)=\frac{(-h)^{r}}{m ! r !} \int_{a}^{b} f^{(r)}(t) B_{r}^{[m-1]}\left(\frac{t-a}{h}-\left\lfloor\frac{t-a}{h}\right\rfloor\right) d t .
$$

Proof. Let $g \in C^{r}[0,1]$. By (4.2) we get

$$
\int_{0}^{1} g(t) d t=\frac{1}{m !} \sum_{k=1}^{r} \frac{(-1)^{k+1}}{k !}\left(g^{(k-1)}(1) B_{k}^{[m-1]}(1)-g^{(k-1)}(0) B_{k}^{[m-1]}\right)+\frac{(-1)^{r}}{m ! r !} \int_{0}^{1} g^{(r)}(t) B_{r}^{[m-1]}(t) d t
$$

Taking $g(t)=f\left(x_{j}+h t\right)$ it is easy to check that $g^{(k)}(t)=h^{k} f^{(k)}\left(x_{j}+h t\right)$ for $k=1,2, \ldots, r$. Substituting $g^{(k-1)}(1), g^{(k-1)}(0), g^{(r)}(t)$ into (4.9), and making a suitable change of variable, we obtain that

$$
\begin{aligned}
\int_{x_{j}}^{x_{j+1}} f(t) d t & =\frac{1}{m !} \sum_{k=1}^{r} \frac{(-1)^{k+1}}{k !} h^{k}\left(f^{(k-1)}\left(x_{j+1}\right) B_{k}^{[m-1]}(1)-f^{(k-1)}\left(x_{j}\right) B_{k}^{[m-1]}\right) \\
& +\frac{(-h)^{r}}{m ! r !} \int_{x_{j}}^{x_{j+1}} f^{(r)}(t) B_{r}^{[m-1]}\left(\frac{t-x_{j}}{h}\right) d t
\end{aligned}
$$

whenever $j=0,1, \ldots, n-1$. Next, adding all these terms for $j=0, \ldots, n-1$ to both sides of (4.10), and nothing that if $x_{j} \leq t \leq x_{j+1}$ then $j \leq \frac{t-a}{h} \leq j+1$, we have

$$
\int_{a}^{b} f(t) d t=\sum_{j=0}^{n-1} \int_{x_{j}}^{x_{j+1}} f(t) d t=\frac{1}{m !} \sum_{j=0}^{n-1} \sum_{k=1}^{r} \frac{(-1)^{k+1}}{k !} h^{k}\left(f^{(k-1)}\left(x_{j+1}\right) B_{k}^{[m-1]}(1)-f^{(k-1)}\left(x_{j}\right) B_{k}^{[m-1]}\right)+R_{r}^{[m-1]}(f) .
$$

From this last equation (4.8) follows.

We conclude this section with a result that reveals an interesting property about the applications of the quadrature formulae of Euler-Maclaurin type (4.8). Using the approach given in [11, pp. 117-120], it is possible to provide a theorem comparing simultaneously the convergence of a series $\sum_{k=1}^{\infty} f(k)$ and an integral $\int_{1}^{\infty} f(x) d x$ in the setting of generalized Bernoulli polynomials of level $m$. In particular, with such a theorem we can estimate the values $\zeta(2 k+1)$, for $k \geq 1$.

Let $r \geq 1, f \in C^{r}[1, \infty)$. For a fixed $m \in \mathbb{N}$, we will denote by

$$
\begin{aligned}
& S(l):=\sum_{j=1}^{l} f(j), \\
& \tilde{\sigma}_{r}^{[m-1]}\left(q_{1}\right):=f\left(q_{1}\right)+\frac{1}{m !} \sum_{k=1}^{r} \frac{(-1)^{k+1}}{k !} f^{(k-1)}\left(q_{1}\right) B_{k}^{[m-1]},
\end{aligned}
$$




$$
\begin{aligned}
\sigma_{r}^{[m-1]}\left(q_{2}\right) & :=\frac{1}{m !} \sum_{k=1}^{r} \frac{(-1)^{k+1}}{k !} f^{(k-1)}\left(q_{2}\right) B_{k}^{[m-1]}(1), \\
\rho_{r}^{[m-1]}\left(q_{1}, q_{2}\right) & :=\frac{1}{m !} \sum_{j=q_{1}+1}^{q_{2}-1} \sum_{k=2}^{r} \frac{(-1)^{k+1}}{k !}\left(B_{k}^{[m-1]}(1)-B_{k}^{[m-1]}\right) f^{(k-1)}(j), \\
R_{r}^{[m-1]}\left(q_{1}, q_{2}\right) & :=\frac{(-1)^{r}}{r !} \int_{q_{1}}^{q_{2}} g_{r}^{[m-1]}(t) d t,
\end{aligned}
$$

where $l, q_{1}, q_{2} \in \mathbb{N}$ and $g_{r}^{[m-1]}(t)=f^{(r)}(t) B_{r}^{[m-1]}(t-\lfloor t\rfloor)$. As well as, we will consider the following limits:

$$
\begin{aligned}
S(\infty) & :=\lim _{l \rightarrow \infty} S(l), \\
\sigma_{r}^{[m-1]}(\infty) & :=\lim _{q_{2} \rightarrow \infty} \sigma_{r}^{[m-1]}\left(q_{2}\right), \\
\rho_{r}^{[m-1]}\left(q_{1}, \infty\right) & :=\lim _{q_{2} \rightarrow \infty} \rho_{r}^{[m-1]}\left(q_{1}, q_{2}\right), \\
R_{r}^{[m-1]}\left(q_{1}, \infty\right) & :=\lim _{q_{2} \rightarrow \infty} R_{r}^{[m-1]}\left(q_{1}, q_{2}\right), \\
e_{r}^{[m-1]}\left(q_{1}\right) & :=\rho_{r}^{[m-1]}\left(q_{1}, \infty\right), \\
\delta_{r}^{[m-1]}\left(q_{1}\right) & :=R_{r}^{[m-1]}\left(q_{1}, \infty\right) .
\end{aligned}
$$

For the reader's convenience, we recall the definition of Euler's constant for a function $f$ (cf. [11, p. 118]). For $f \in C^{r}[1, \infty)$ and any $n \in \mathbb{N}$ let us consider the sequence

$$
\gamma_{n}(f):=\sum_{i=1}^{n} f(i)-\int_{1}^{n} f(t) d t .
$$

Euler's constant for function $f$ is defined as the limit

$$
\gamma(f):=\lim _{n \rightarrow \infty} \gamma_{n}(f)
$$

whenever such limit exists and be finite.

The quadrature formulae of Euler-Maclaurin type (4.8) is also of theoretical interest. More precisely, the definitions (4.16), (4.17) and the formulae (4.8) imply the following result:

Theorem 4.3. For a fixed $m \in \mathbb{N}$, every $r, p, n \in \mathbb{N}$ and $f \in C^{r}[1, \infty)$. Assume that $\rho_{r}^{[m-1]}(1, \infty), \int_{1}^{\infty}\left|f^{(r)}(t)\right|$ dt converge, and the finite limit $\lambda_{0}:=\lim _{n \rightarrow \infty} f(n)$ exists, then

(a) The integral $\int_{1}^{\infty} f(t) d t$ converges if and only if the series $\sum_{j=1}^{\infty} f(j)$ converges.

(b) If the integral $\int_{1}^{\infty} f(t) d t$ converges, then

$$
\int_{1}^{\infty} f(t) d t=\int_{1}^{p} f(t) d t+S(\infty)-S(p-1)+\sigma_{r}^{[m-1]}(\infty)-\tilde{\sigma}_{r}^{[m-1]}(p)+e_{r}^{[m-1]}(p)+\delta_{r}^{[m-1]}(p) .
$$

Notice that if $\rho_{r}^{[m-1]}(1, \infty)$ converges, then $\lim _{n \rightarrow \infty} f^{(k-1)}(n)=0$ for every $k=2, \ldots, r$.

Proof. Without loss of generality we can assume that $p \leq n$. The substitution $a=p, b=n$ and $h=1$ into (4.8) and the use of (4.11)-(4.15) yield the identity

$$
\int_{p}^{n} f(t) d t=S(n-1)-S(p-1)-f(n)+\sigma_{r}^{[m-1]}(n)-\widetilde{\sigma}_{r}^{[m-1]}(p)+\rho_{r}^{[m-1]}(p, n)+R_{r}^{[m-1]}(p, n)
$$

where $S(0)=0$ by definition. The remainder $R_{r}^{[m-1]}(p, n)$ can be estimated by

$$
\left|R_{r}^{[m-1]}(p, n)\right| \leq \frac{\mu_{r}^{[m-1]}}{r !} \int_{p}^{n}\left|f^{(r)}(t)\right| d t,
$$

where $\mu_{r}^{[m-1]}=\max \left\{\left|B_{r}^{[m-1]}(x)\right|: 0 \leq x \leq 1\right\}$.

By (4.16) and the formula (4.18) we obtain

$$
\gamma_{n}(f)=f(n)+\widetilde{\sigma}_{r}^{[m-1]}(1)-\sigma_{r}^{[m-1]}(n)-\rho_{r}^{[m-1]}(1, n)-R_{r}^{[m-1]}(1, n)
$$

Our assumptions imply, according to (4.20), that the Euler's constant for the function $f, \gamma(f)$, exists and the next equality is satisfied:

$$
\gamma(f)=\lambda_{0}+\widetilde{\sigma}_{r}^{[m-1]}(1)-\sigma_{r}^{[m-1]}(\infty)-\rho_{r}^{[m-1]}(1, \infty)-R_{r}^{[m-1]}(1, \infty)
$$


Now, from (4.20) and (4.21) we have

$$
\gamma(f)=\gamma_{n}(f)+\lambda_{0}-f(n)+\sigma_{r}^{[m-1]}(n)-\sigma_{r}^{[m-1]}(\infty)-e_{r}^{[m-1]}(n-1)-\delta_{r}^{[m-1]}(n),
$$

where

$$
\left|\delta_{r}^{[m-1]}(n)\right| \leq \frac{\mu_{r}^{[m-1]}}{r !} \int_{n}^{\infty}\left|f^{(r)}(t)\right| d t .
$$

Thus, substituting (4.16) into (4.22) and using (4.11) we obtain

$$
\int_{1}^{n} f(t) d t=S(n)-\gamma(f)+\lambda_{0}-f(n)+\sigma_{r}^{[m-1]}(n)-\sigma_{r}^{[m-1]}(\infty)-e_{r}^{[m-1]}(n-1)-\delta_{r}^{[m-1]}(n) .
$$

Finally, part $(a)$ of Theorem 4.3 can be deduced from (4.23). In order to obtain part $(b)$ of Theorem 4.3 it suffices to consider (4.18) and the equality $\int_{p}^{n} f(t) d t=\int_{1}^{n} f(t) d t-\int_{1}^{p} f(t) d t$.

The interested reader may consult the analogous result for $m=1$ in [11, Theorem 2].

Example 4.4. To compute $\zeta(3)=\sum_{k=1}^{\infty} \frac{1}{k^{3}}$, we can put $m=5, r=2, p=100, f(x)=\frac{1}{x^{3}}, x \in[1, \infty)$ and apply part $(b)$ of Theorem 4.3. Then, we obtain

$$
\begin{aligned}
S(99) & =1.2020064006596776104, \\
\sigma_{2}^{[4]}(p) & =\frac{5}{6 p^{3}}+\frac{85}{84 p^{4}}, \\
\sigma_{2}^{[4]}(100) & =8.4345238095238095238 \times 10^{-7}, \\
\sigma_{2}^{[4]}(\infty) & =0 \\
\tilde{\sigma}_{2}^{[4]}(p) & =\frac{5}{6 p^{3}}+\frac{1}{84 p^{4}}, \\
\tilde{\sigma}_{2}^{[4]}(100) & =8.3345238095238095238 \times 10^{-7}, \\
e_{2}^{[4]}(100) & =3.2836666500022217224 \times 10^{-7} .
\end{aligned}
$$

Next, part (b) of Theorem 4.3 gives

$$
\begin{aligned}
\zeta(3) & =\int_{100}^{\infty} \frac{d t}{t^{3}}+S(99)-\sigma_{2}^{[4]}(\infty)+\widetilde{\sigma}_{2}^{[4]}(100)-e_{2}^{[4]}(100)-\delta_{2}^{[4]}(100) \\
& =0.00005+1.2020064006596776104+(8.3345238095238095238) \times 10^{-7} \\
& -(3.2836666500022217224) \times 10^{-7}-\delta_{2}^{[4]}(100) \\
& =1.2020560622930126102-\delta_{2}^{[4]}(100) .
\end{aligned}
$$

Since

$$
\delta_{2}^{[4]}(100) \approx 3.10296 \times 10^{-7},
$$

we obtain the following estimates for $\zeta(3)$ :

$$
\zeta(3) \approx 1.2020557519970993510 \text {. }
$$

In this case, our approximation is accurate up to five decimal places of $\zeta(3)=1.2020569031595942854 \ldots .$. Since, for $p \geq 1$,

$$
\left|\delta_{2}^{[4]}(p)\right| \leq \frac{\mu_{2}^{[4]}}{2} \int_{p}^{\infty} \frac{12}{t^{5}} d t=\frac{850}{7 p^{4}} .
$$

Then,

$$
\left|\delta_{2}^{[4]}(100)\right| \leq 0.000001214285714,
$$

and the estimate (4.24) could be refined in order to get an accurate up to six decimal places. 
Example 4.5. Now, we will estimate $\zeta(3)=\sum_{k=1}^{\infty} \frac{1}{k^{3}}$, taking $m=2, r=2, p=20, f(x)=\frac{1}{x^{3}}, x \in[1, \infty)$ and apply part (b) of Theorem 4.3 again. In this case, we have

$$
\begin{aligned}
S(19) & =1.2020064006596776104, \\
\sigma_{2}^{[1]}(p) & =\frac{2}{3 p^{3}}+\frac{7}{12 p^{4}}, \\
\sigma_{2}^{[1]}(20) & =0.000086979166666666666667, \\
\sigma_{2}^{[1]}(\infty) & =0 \\
\tilde{\sigma}_{2}^{[1]}(p) & =\frac{2}{3 p^{3}}+\frac{1}{12 p^{4}}, \\
\tilde{\sigma}_{2}^{[1]}(20) & =0.000083854166666666666667, \\
e_{2}^{[1]}(20) & =0.00002244785177830327
\end{aligned}
$$

From part (b) of Theorem 4.3 we get

$$
\begin{aligned}
\zeta(3) & =\int_{20}^{\infty} \frac{d t}{t^{3}}+S(19)-\sigma_{2}^{[1]}(\infty)+\widetilde{\sigma}_{2}^{[1]}(20)-e_{2}^{[1]}(20)-\delta_{2}^{[1]}(20) \\
& =0.00125+1.2007428419584369581+0.000083854166666666666667-0.00002244785177830327-\delta_{2}^{[1]}(20) \\
& =1.2020560522930126102-\delta_{2}^{[1]}(20) .
\end{aligned}
$$

Since

$$
\delta_{2}^{[1]}(20) \approx 9.40 \times 10^{-7},
$$

we obtain the following numerical approximation of $\zeta(3)$

$$
\zeta(3) \approx 1.2019663288791965826,
$$

which only is accurate up to two decimal places of $\zeta(3)=1.2020569031595942854 \ldots$.

Example 4.6. To estimate $\zeta(5)=\sum_{k=1}^{\infty} \frac{1}{k^{5}}$, we put $m=2, r=6, p=30, f(x)=\frac{1}{x^{5}}, x \in[1, \infty)$ and apply part (b) of Theorem 4.3. In this case, we have

$$
\begin{aligned}
S(29) & =1.0369274253541474188, \\
\sigma_{6}^{[1]}(p) & =\frac{2}{3 p^{5}}+\frac{35}{36 p^{6}}+\frac{8}{9 p^{7}}+\frac{77}{216 p^{8}} \\
& -\frac{26}{81 p^{9}}-\frac{151}{270 p^{10}}, \\
\sigma_{6}^{[1]}(30) & =2.8809650704405569010 \times 10^{-8}, \\
\sigma_{6}^{[1]}(\infty) & =0, \\
\tilde{\sigma}_{6}^{[1]}(p) & =\frac{2}{3 p^{5}}+\frac{5}{36 p^{6}}+\frac{1}{18 p^{7}}-\frac{7}{216 p^{8}} \\
& -\frac{5}{81 p^{9}}-\frac{1}{270 p^{10}}, \\
\tilde{\sigma}_{6}^{[1]}(30) & =2.7627849714267435143 \times 10^{-8}, \\
e_{6}^{[1]}(30) & =6.48060252152 \times 10^{-9} .
\end{aligned}
$$

From part (b) of Theorem 4.3 we get

$$
\begin{aligned}
\zeta(5) & =\int_{30}^{\infty} \frac{d t}{t^{5}}+S(29)-\sigma_{3}^{[1]}(\infty)+\widetilde{\sigma}_{3}^{[1]}(30)-e_{3}^{[1]}(30)-\delta_{3}^{[1]}(30) \\
& =3.0864197530864197531 \times 10^{-7}+1.0369274253541474188+2.7627902250673169740 \times 10^{-8} \\
& -6.47839130112 \times 10^{-9}-\delta_{6}^{[1]}(30) \\
& =1.0369277263337192158-\delta_{6}^{[1]}(30) .
\end{aligned}
$$

According to

$$
\delta_{6}^{[1]}(30) \approx-3.9236379933251 \times 10^{-51},
$$

we obtain the following numerical approximation of $\zeta(5)$

$$
\zeta(5) \approx 1.0369277263337192158 .
$$

So, our approximation is accurate up to seven decimal places of $\zeta(5)=1.0369277551433699263 \ldots$. 
In [11, Example 5] the examples 4.4 and 4.5 are considered for the level $m=1$. Indeed, putting $r=2$ and $p=20$ the estimate (4.24) is also obtained. So, from a numerical viewpoint the level $m=1$ seems to provide a low computational cost.

Finally, the numerical evidence corresponding to the examples 4.4-4.6 suggests that when $m>1$ for obtaining higher precision for our approximations to the series $\sum_{j=1}^{\infty} f(j)$ we need only use higher values of $r$ in part (b) of Theorem 4.3.

Example 4.7. Using part (a) of Theorem 4.3 we can deduce that the series

$$
\operatorname{sum}_{k=1}^{\infty} \frac{\cos (\sqrt{k})}{k}
$$

converges, since

$$
\int_{1}^{\infty} \frac{\cos (\sqrt{t})}{t} d t \approx-0.67480784580193626932 \ldots
$$

The above approximation was performed using MAPLE 15. However, it is not difficult to show that the integral $\int_{1}^{\infty} \frac{\cos (\sqrt{t})}{t} d t$ converges. Notice that

$$
2 \int_{1}^{b} \frac{d(\sin (\sqrt{t}))}{\sqrt{t}}=\int_{1}^{b} \frac{\cos (\sqrt{t})}{t} d t
$$

and by the formula for integration by parts of Riemann-Stieltjes, we have:

$$
2\left[\int_{1}^{b} \frac{d(\sin (\sqrt{t}))}{\sqrt{t}}+\int_{1}^{b} \sin (\sqrt{t}) d\left(\frac{1}{\sqrt{t}}\right)\right]=2\left(\frac{\sin (\sqrt{b})}{\sqrt{b}}-\sin (1)\right) .
$$

Consequently,

$$
\int_{1}^{b} \frac{\cos (\sqrt{t})}{t} d t=2\left(\frac{\sin (\sqrt{b})}{\sqrt{b}}-\sin (1)\right)+\int_{1}^{b} \frac{\sin (\sqrt{t})}{t^{3 / 2}} d t
$$

since $\lim _{b \rightarrow \infty} \frac{\sin (\sqrt{b})}{\sqrt{b}}=0$ and the integral $\int_{1}^{\infty} \frac{\sin (\sqrt{t})}{t^{3 / 2}} d t$ converges, then

$$
\int_{1}^{\infty} \frac{\cos (\sqrt{t})}{t} d t \quad \text { converges. }
$$

We can provide another solution by using Dirichlet's test for improper integrals (see for instance, [11, Example 4] where a similar series is considered.)

\section{Acknowledgement}

Research partially supported by Decanato de Investigación y Desarrollo, Universidad Simón Bolívar, Venezuela, grant DID-USB (S1-IC-CB004-17).

\section{References}

[1] T. M. Apostol, Another elementary proof of Euler's formula for $\zeta(2 n)$, AM. Math. Monthly, 80 (1973), $425-431$

[2] T. M. Apostol, An elementary view of Euler's summation formula, AM. Math. Monthly, 106 (1999), 409-418.

[3] T. Arakawa, T. Ibukiyama, M. Kaneko, Bernoulli numbers and Zeta Functions, Springer Monographs in Mathematics, Springer, New York, 2014.

[4] R. Ayoub, Euler and the zeta function, AM. Math. Monthly, 81 (1974), 1067-1086.

[5] R. Baker, An Introduction to Riemann's Life, His Mathematics and His Work on the Zeta Function, H. Montgomery, A. Nikeghbali, M. Th. Rassias (editors), Exploring the Riemann Zeta Function: 190 years from Riemann's Birth, Springer International Publishing AG, Switzerland, 2017, pp. 1-12.

[6] B. C. Berndt, A. Straub, Ramanujan's Formula for $\zeta(2 n+1)$, H. Montgomery, A. Nikeghbali, M. Th. Rassias (editors), Exploring the Riemann Zeta Function: 190 years from Riemann's Birth, Springer International Publishing AG, Switzerland, 2017, pp. 13-14.

[7] Ó. Ciaurri, L. M. Navas, F. J. Ruiz, J. L. Varona, A simple computation of $\zeta(2 k)$ by using Bernoulli polynomials and a telescoping series, AM. Math. Monthly, 122 (2015), 444-451.

[8] P. J. Davis, P. Rabinowitz, Methods of Numerical Integration, Academic Press Inc., 1984.

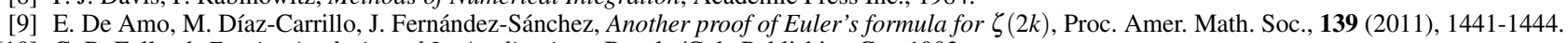

[10] G. B. Folland, Fourier Analysis and Its Applications, Brooks/Cole Publishing Co., 1992.

[11] V. Lampret, The Euler-Maclaurin and Taylor formulas: Twin, elementary derivations, Math. Mag., 74(2) (2001), 109-122.

[12] A. Hassen, H. D. Nguyen, Hypergeometric Bernoulli polynomials and Appell sequences, Int. J. Number Theory, 4(5) (2008), 767-774.

[13] P. Hernández-Llanos, Y. Quintana, A. Urieles, About extensions of generalized Apostol-type polynomials, Results Math., 68 (2015), 203-225.

[14] F. T. Howard, Some sequences of rational numbers related to the exponential function, Duke Math. J., 34 (1967), $701-716$.

[15] P. Natalini, A. Bernardini, A generalization of the Bernoulli polynomials, J. Appl. Math., 2003(3) (2003), 155-163.

[16] N. E. Nørlund, Vorlesungen über Differenzenrechnung, Springer-Verlag, Berlin, 1924, (reprinted 1954), (in German).

[17] G. M. Phillips, Interpolation and Approximation by Polynomials, Springer-Verlag, New York, 2003.

[18] Y. Quintana, W. Ramírez, A. Urieles, On an operational matrix method based on generalized Bernoulli polynomials of level m, Calcolo, 55(3) (2018), 29 pages.

[19] Y. Quintana, A. Urieles, Quadrature formulae of Euler-Maclaurin type based on generalized Euler polynomials of level m, Bull. Comput. Appl. Math., 6(2) (2018), 43-64.

[20] H. M. Srivastava, H. L. Manocha, A Treatise on Generating Functions, Ellis Horwood Ltd., West Sussex, England, 1984.

[21] H. M. Srivastava, J. Choi, Zeta and q-Zeta Functions and Associated Series and Integrals, Elsevier, London, 2012.

[22] H. M. Srivastava, M. Garg, S. Choudhary, A new generalization of the Bernoulli and related polynomials, Russ. J. Math. Phys., 17(2) (2010), 251-261.

[23] R. D. Stuart, Introduction to Fourier Analysis, Methuen \& Co. Ltd., London, 1961. 